\title{
A 3D-Printed Multi-Chamber Device Allows Culturing Cells On Buckypapers Coated With PAMAM Dendrimer And Obtain Innovative Materials For Biomedical Applications
}

This article was published in the following Dove Press journal:

International Journal of Nanomedicine

\begin{abstract}
Alessandro Paolini ${ }^{1}$
Giulia Battafarano (D)

Valentina D'Oria'

Francesco Mura (iD ${ }^{2}$

Simona Sennato (D) $^{3}$

Valentina Mussi ${ }^{4}$

Roberta Risoluti (iD) 5

Stefano Materazzi (iD ${ }^{5}$

Andrea Del Fattore ${ }^{\prime}$

Andrea Masotti ${ }^{1}$
\end{abstract}

'Bambino Gesù Children's Hospital, IRCCS, Research Laboratories, Rome 00I 46, Italy; ${ }^{2}$ Center for Nanotechnology for Engineering (CNIS), Sapienza University of Rome, Rome 00 185, Italy; ${ }^{3}$ CNR-ISC UOS Sapienza and Physics Department, Sapienza University of Rome, Rome 00185, Italy; ${ }^{4}$ National Research Council, Institute for Microelectronics and Microsystems IMMCNR, Roma 00133, Italy; ${ }^{5}$ Department of Chemistry, Sapienza University of Rome, Rome 00185, Italy
Correspondence: Andrea Masotti Bambino Gesù Children's Hospital, IRCCS, Research Laboratories, Gene Expression-Microarrays Laboratory, Viale di San Paolo 15, Rome 00I46, Italy

Tel +39-06-68592650

Fax +39-0668592904

Email andrea.masotti@opbg.net
Background: The advent of 3D printing technology allowed the realization of custom devices that can be used not only in the everyday life but also in the nanotechnology and biomedical fields. In nanotechnology, the use of bi-dimensional nanostructures based on carbon nanotubes, generally referred as buckypapers, have received considerable attention for their versatility and potential application in many biomedical fields. Unfortunately, buckypapers are extremely hydrophobic and cannot be used in aqueous media to culture cells.

Methods: A polymeric device able to accommodate buckypapers and facilitate cell growth was fabricated by using 3D printing technology. We imparted hydrophilicity to buckypapers by coating them with polyamidoamine (PAMAM) dendrimers.

Results: We found that by using novel techniques such as polymer coating the buckypaper hydrophilicity increased, whereas the use of 3D printing technology allowed us to obtain custom devices that have been used to culture cells on buckypapers for many days. We characterized in details the morphology of these structures and studied for the first time the kinetic of cell proliferation. We found that these scaffolds, if properly functionalized, are suitable materials to grow cells for long time and potentially employable in the biomedical field.

Conclusion: Although these materials are cytotoxic under certain circumstances, we have found a suitable coating and specific experimental conditions that encourage using buckypapers as novel scaffolds for cell growth and for potential applications in tissue repair and regeneration. Keywords: 3D printing, buckypaper, PAMAM dendrimer, cell proliferation, tissue regeneration, transfection

\section{Introduction}

In the last decades, single- and multi-walled carbon nanotubes (SWCNTs and MWCNTs, respectively) have been extensively studied for their numerous electrical and physical properties and have been applied in many biomedical applications (i.e., drug delivery and diagnostic devices). ${ }^{1-4}$ Carbon nanofibers, carboxylated CNTs, and other functionalized CNTs have been demonstrated to increase the growth, spreading, and adhesion of human osteoblasts (CRL-11372), mouse fibroblasts (L929), and hippocampal neurons, suggesting that CNTs could be an ideal starting material for the generation of scaffolds/matrices. ${ }^{5-7}$ However, the concept of using bidimensional assemblies of CNTs, generally referred as "buckypaper" (BP), as suitable cell culture substrates for tissue engineering has been only marginally considered. ${ }^{8}$ In fact, the most 
commonly used materials for the production of 3D scaffolds are biodegradable synthetic polymers such as poly-(L-lactic acid), poly-(glycolic acid), poly-caprolactone, or biopolymers such as collagen and fibroin. ${ }^{9}$

Many researchers focused their efforts on studying the interactions between CNTs and living mammalian cells, but in tissue engineering, these interactions are crucial to apply artificial 3D scaffolds in practice. ${ }^{10}$ Engineered scaffolds provide a synthetic extracellular matrix (ECM) able not only to support the adhesion, proliferation, and migration of cells but also to guide tissue regeneration by the host or other transplanted cells. The microstructure of 3D scaffolds and the surface properties of CNTs are also key factors to determine cell adhesion, proliferation, and migration. $^{7,11}$

Aimed at using CNTs BP as innovative substrate for biomedical applications, we had to solve the problem of the intrinsic hydrophobicity of BP that prevented any use of aqueous solutions, such as culture media. Nowadays, 3D technology allows to prototype custom 3D objects by progressive deposition of fused material ${ }^{12,13}$ allowing researchers to produce their own custom-made research tools (i.e., micropipettes, micromanipulators, syringe pumps, webcam-based microscopes, smartphone holder, and time-lapse cells incubators). ${ }^{14-17}$ Therefore, by following an established procedure, ${ }^{1,2}$ we coated BP with a polyamidoamine polymer (PAMAM) and transformed its surface from hydrophobic to hydrophilic, whereas by exploiting the 3D printing technology, we designed and created a custom device able to let grow cells on BP surface for many days and to study its physicochemical properties for possible biomedical applications. Finally, to add further functionality to this scaffold, we assessed the ability of coated-BP to deliver a microRNA mimic and investigated the long-term delivery of these RNA molecules to the cell layer.

\section{Materials And Methods \\ Materials}

Commercial MWCNT BP was purchased from Buckeye Composites (Kettering, USA), poly-amidoamine dendrimer generation 5 (PAMAM G=5, cat.no. 536709), RIPA buffer (cat.no R0278) and FAM-Pre-mir-503 oligonucleotide (batch no. HA08019806) were purchased from Sigma-Aldrich and used as received. Pierce ${ }^{\mathrm{TM}}$ BCA protein assay kit (cat.no. 23227) and Halt ${ }^{\mathrm{TM}}$ phosphatase inhibitor cocktail (cat.no 78420) were purchased from ThermoFisher Scientific.
Polyethylene terephthalate glycol-modified (PETG) filaments were purchased from Galaxy Stampanti 3D (Mantova, Italy).

\section{Ethical Approval For Use Of Human Cell Lines And Tissues}

The Ethical committee of Bambino Gesù Children's Hospital approved the study and the use of human cells for this project. Written informed consent was provided by donors. Patient's data were also anonymized and treated with confidentiality.

\section{Cell Cultures}

Human embryonic kidney 293 (HEK-293T) (ATCC $^{\circledR}$ CRL1573) and human osteosarcoma (Saos-2) cells (ATCC ${ }^{\circledR}$ HTB85) were maintained in DMEM high glucose medium (Euroclone), supplemented with $10 \%$ fetal bovine serum (FBS; Euroclone), $50 \mathrm{U} / \mathrm{mL}$ penicillin (Euroclone), and 50 $\mu \mathrm{g} / \mathrm{mL}$ streptomycin (Euroclone) at $37^{\circ} \mathrm{C}$ in $5 \% \mathrm{CO}_{2}$. To obtain osteoclasts, peripheral blood mononuclear cells (PBMCs) were isolated from citrate blood samples of healthy donors by density-gradient centrifugation in Ficoll (Histopaque 1077 Human Lymphocyte, Sigma Aldrich). The pellet was rinsed with PBS and re-suspended in DMEM essential medium (Euroclone) containing $100 \mathrm{U} / \mathrm{mL}$ penicillin, $100 \mathrm{mg} / \mathrm{mL}$ streptomycin (Euroclone), $2 \mathrm{mM} \mathrm{L-glutamine}$ (Euroclone), and 10\% heat-inactivated fetal bovine serum (FBS, Gibco). Cells were seeded at a density of $2 \times 10^{6}$ cells/ $\mathrm{mL}$ on a T75 flask and cultured in $5 \% \mathrm{CO}_{2}$ atmosphere. After overnight incubation, the cultures were supplemented with recombinant human M-CSF (25 ng/mL; Peprotech). After reaching $80 \%$ confluence, cells were treated with recombinant human RANK-L (30 ng/mL; Peprotech) and M-CSF for 14 days. Medium and factors were replaced every 3-4 days.

\section{BP Coating With PAMAM}

Small round pieces of BP with a diameter of approximately $1 \mathrm{~cm}(\sim 3 \mathrm{mg}$ each) were cut from a commercial sheet of carbon BP. For PAMAM coating, BP was covered with distilled water $(0.3 \mathrm{~mL})$ containing PAMAM at a final concentration of $10 \mathrm{mg} / \mathrm{mL}$. BP was gently mixed for 30 mins at room temperature then placed for 3 days at $+4^{\circ} \mathrm{C}$. At the end of incubation, both the PAMAM-coated buckypaper (PAM-BP) and the pristine BP were washed in PBS and used for all of the downstream experiments.

\section{Atomic-Force Microscopy (AFM)}

AFM measurements were performed with a Dimension Icon (Bruker AXS) instrument. AFM images were 
acquired in air at room temperature and under ambient conditions, by employing Tapping Mode. We used RTESP-300 (Brucker) probes characterized by a sharp silicon tip (nominal radius of curvature $10 \mathrm{~nm}$ ), mounted on a silicon nitride rectangular cantilever with nominal resonant frequency of $300 \mathrm{kHz}$ and nominal spring constant of $40 \mathrm{~N} / \mathrm{m}$. Samples were stuck on the AFM sample support by using double-sided tape $1 \mathrm{hr}$ before the measurement to allow sample stabilization and avoid mechanical noise. Images were analyzed using Nanoscope Analysis 1.5 software (Bruker). Height Sensor and Phase Channels were presented as raw data, except for flattening applied to height sensor channel.

\section{Thermogravimetric Analysis}

TGA curves were recorded using a TGA7 equipment (PerkinElmer Inc., Waltham, MA, USA). The samples investigated were heated in platinum crucibles in the temperature range $20-850^{\circ} \mathrm{C}$, in pure nitrogen or air atmosphere (gaseous mixture of nitrogen and oxygen with $80 \%$ and $20 \%$, v/v, respectively) under a flow rate of $100 \mathrm{~mL} / \mathrm{min}$. The scanning rate for the best resolution was found to be $10^{\circ} \mathrm{C} / \mathrm{min}$. To ensure an accurate measurement of sample temperature, the calibration was performed using the Curie-point transition of standard metals, according to manufacturer's recommendations. Each sample was analyzed in triplicate.

\section{Raman Characterization}

Raman spectra were acquired with a DXR Thermo Fisher Scientific Raman Microscope, by exciting the samples at $532 \mathrm{~nm}$ with $8 \mathrm{~mW}$ laser power and a $100 \times$ objective. The measured spectral range was $150-3400 \mathrm{~cm}^{-1}$ and each spectrum resulted from $0.8 \mathrm{~s}$ acquisition time and 120 accumulations over an area of about $800 \mathrm{~nm}$ (laser spot).

\section{D Printing Of A Custom Device For Cell Culture}

BP is highly hydrophobic (see Figure S1), and small pieces of BP in aqueous solutions (i.e., cell cultures) tend to float. In order to prevent this phenomenon and maintain BP stuck to the bottom of the culture container, a custom multi-chamber device was 3D-printed by using a 3DRAG printer (Futura Group srl (C), Italy) using a PETG filament. An extrusion temperature of $220^{\circ} \mathrm{C}$ and a bed temperature of $60^{\circ} \mathrm{C}$ have been set to print the 3D device. PETG is more resistant than poly-lactide (PLA, poly-lactic acid) filaments and combines the benefits of Acrylonitrile
Butadiene Styrene (ABS) filaments (robustness and temperature resistance) to that of PLA (ease of printing and recyclability). Moreover, PETG 3D-printed objects can be easily sterilized in an autoclave. The device was designed using the Thinkercad app (www.tinkercad.com, (C) 2016 Autodesk, Inc.), a browser-based 3D design and modeling tool, with the concept to enclose the BP within the structure and separately confine cells into cylindrical chambers.

\section{Cell Culture On BP}

Many BP pieces were mounted in the 3D-printed multichamber device after enclosing the tiny sheets between two silicon rings. HEK-293T cells and Saos-2 cells were plated at a density of $6 \times 10^{4} / \mathrm{cm}^{2}$, whereas osteoclasts at a density of $8 \times 10^{4} / \mathrm{cm}^{2}$. In each chamber of the 3D-printed device, $3 \mathrm{~mL}$ of growth medium were added. At the end of the culture, the 3D-printed device was disassembled and the BP washed once with PBS before processing.

\section{Scanning Electron Microscopy And Energy Dispersive X-Ray Characterization}

Scanning electron microscopy was employed to visualize the amount and the morphology of cells grown on BPs. Both field emission scanning electron microscopy (FESEM) and energy dispersive $\mathrm{X}$-ray (EDX) analysis were performed on $\mathrm{BP}$ and PAM-BP using the Zeiss Auriga electron microscope equipped with a Bruker Quantax detector (Energy resolution $123 \mathrm{eV}$ for Mn K $\alpha$ line). Cells were grown on BP and PAMBP for several days (i.e., 1, 3, and 7 days for HEK-293T and Saos-2 and 3 days for osteoclasts) and finally fixed with $2.5 \%$ glutaraldehyde in $0.15 \mathrm{M}$ cacodylate buffer ( $\mathrm{pH} 7.2$ ) for $2 \mathrm{hrs}$ at room temperature. After a final dehydration step in graded alcohol, the BPs were examined after coating them with 30 $\mathrm{nm}$ of Chromium using a Quorum 150T sputter. We operated a low acceleration voltage and current in order to avoid damaging cells.

\section{BCA Protein Assay}

To assess cell proliferation on carbon BPs, we employed the Pierce BCA protein assay. To evaluate the reliability of the test, HEK-293T and Saos-2 cells were cultured on BP and PAM-BP for 1, 3, and 7 days. Then, the medium was removed and cells rinsed with PBS at room temperature. Proteins were extracted by adding $50 \mu \mathrm{L}$ of ice-cold RIPA buffer with $1 \mathrm{x}$ Halt phosphatase inhibitor cocktail to each BP and incubated on ice for 5 mins. The lysate was centrifuged at $15,000 \times \mathrm{g}$ for 15 mins at $4^{\circ} \mathrm{C}$. The protein concentration was measured by 
BCA protein assay kit. Control samples consisting of the same number of cells seeded on BP were lysed and the protein concentration was immediately measured.

\section{Confocal Microscopy}

To study the ability of BPs to transfect cells, $50 \mathrm{nM}$ of FAMpre-mir-503 was incubated with BP and PAM-BP at a BP/ oligonucleotide ratio of 20:1 (w/w) for $15 \mathrm{mins}$ at room temperature to allow complex formation. ${ }^{1,2}$ After 1, 3 and 7 days, HEK-293T and Saos-2 cells were washed once with cold phosphate-buffered saline (PBS) and fixed by incubation with a paraformaldehyde $4 \%$ solution for 15 mins at room temperature. Cells were stained with a phalloidinTRITC/PBS solution (1:100) for $1 \mathrm{hr}$, washed again and the nuclei stained with the intercalating dye Hoechst 33258/PBS solution (1:2500) for 3 mins. Fixed cells were mounted with a glycerol/PBS solution (3:1) and kept covered to prevent dye photobleaching. To observe the transfection by PAM-BP, pristine BPs were functionalized with a PAMAM derivative previously labeled with rhodamine $\mathrm{B}$ isothiocyanate (RhoPAM). ${ }^{18}$ After 3 days of incubation with Rho-PAM-BP, osteoclasts were observed using the same protocol described for HEK-293T cells, after incubation with phalloidin-FITC/ PBS solution (1:100) for 45 mins. Images were acquired with an Olympus Fluoview FV1000 confocal microscope (Olympus, Italy) equipped with FV10-ASW version 2.0 software, Multi Ar (458-488 and 512 nm), 2X He/Ne (543 and $633 \mathrm{~nm}$ ), and 405-nm diode laser, 60X magnification.

\section{Statistical Analysis}

Data were obtained from three independent experiments. Data were expressed as means with standard error of means and were analyzed by using Student's $t$-test employing SPSS (ver.20) software. A p-value $<0.05$ was considered statistically significant.

\section{Results}

\section{PAMAM Dendrimer Binds BP Stably And Imparts Roughness To The Surface}

The morphology of both BP and PAM-BP was analyzed by scanning electron microscopy. SEM images of BP and PAM-BP showed multiple interlaced layers of CNTs of various lengths. The depth of CNTs multilayer organization is represented by the darkest parts of the imaged layer (Figure 1A). PAMAM functionalization was visible as a thin film covering CNTs. By SEM this coating appeared as a "veil" that covered all of the surface resulting in a light gray appearance of the layer (Figure 1B).

To confirm the interaction between the BP and the PAMAM dendrimer, we performed an EDX analysis. The EDX analysis of BP indicated the presence of a high percentage of carbon $(\mathrm{C}=98.56 \%)$, small traces of oxygen $(\mathrm{O}=0.59 \%)$, and impurities of iron $(\mathrm{Fe}=0.85 \%)$ likely due to the catalyzed preparation of CNTs (Figure 1C).

After PAMAM functionalization, the EDX analysis of PAM-BP showed the presence of nitrogen $(\mathrm{N}=7.38 \%)$ and an increased amount of oxygen $(\mathrm{O}=4.62 \%)$, confirming the interaction between the dendrimer and the BP surface (Figure 1D).

To better characterize the interaction of PAMAM dendrimers with the BP, samples were visualized also by AFM. The possibility to have topographical images with high magnification allowed us to explore in detail the sample surface and confirm the coating of BP by PAMAM as already observed by SEM microscopy. In fact, the surface of pristine BP appeared smooth and well defined both in height (Figure 2A) and phase image (Figure 2B). In Figure 2C, the appearance of PAMAMcoated BP was similar to that of BP, whereas the phase channel was able to reveal a wrinkled surface due to the presence of "bubble-like" polymer on the nanotube surface (the small structures marked by arrows in Figure 2D) that imparted a jagged appearance to the PAM-BP surface.

TGA analysis confirmed the presence of PAMAM $(9.34 \% \pm 1.07 \%)$ absorbed onto the BP surface. Compared to the absorption that we observed in our previous work, ${ }^{2}$ where we prepared PAMAM-coated CNTs, the percentage of absorption was significantly lower. In fact, we found that for PAM-CNTs the amount of polymer absorbed was $21.36 \% \pm 3.57 \%$, more than the double of what was found for PAM-BP. This was likely due to the higher surface area of monodimensional CNTs compared to that of bidimensional BP. In fact, most of the inner CNTs are not exposed to the PAMAM solution.

To investigate the binding ability of PAMAM toward BP and analyze the physicochemical modifications induced by the functionalization, PAM-BP samples were also characterized by Raman spectroscopy (Figure 3). All the typical bands of the BP can be recognized in the spectra reported in Figure 3A, collected on the sample before (black curve) and after (red curve) PAMAM treatment. In fact, the spectral features with higher intensities can be identified as the $G$ Band (at about $1574 \mathrm{~cm}^{-1}$ ) ascribed to the graphitic nature of the material, the $\mathrm{D}$ band (at about $1343 \mathrm{~cm}^{-1}$ ) related to the 

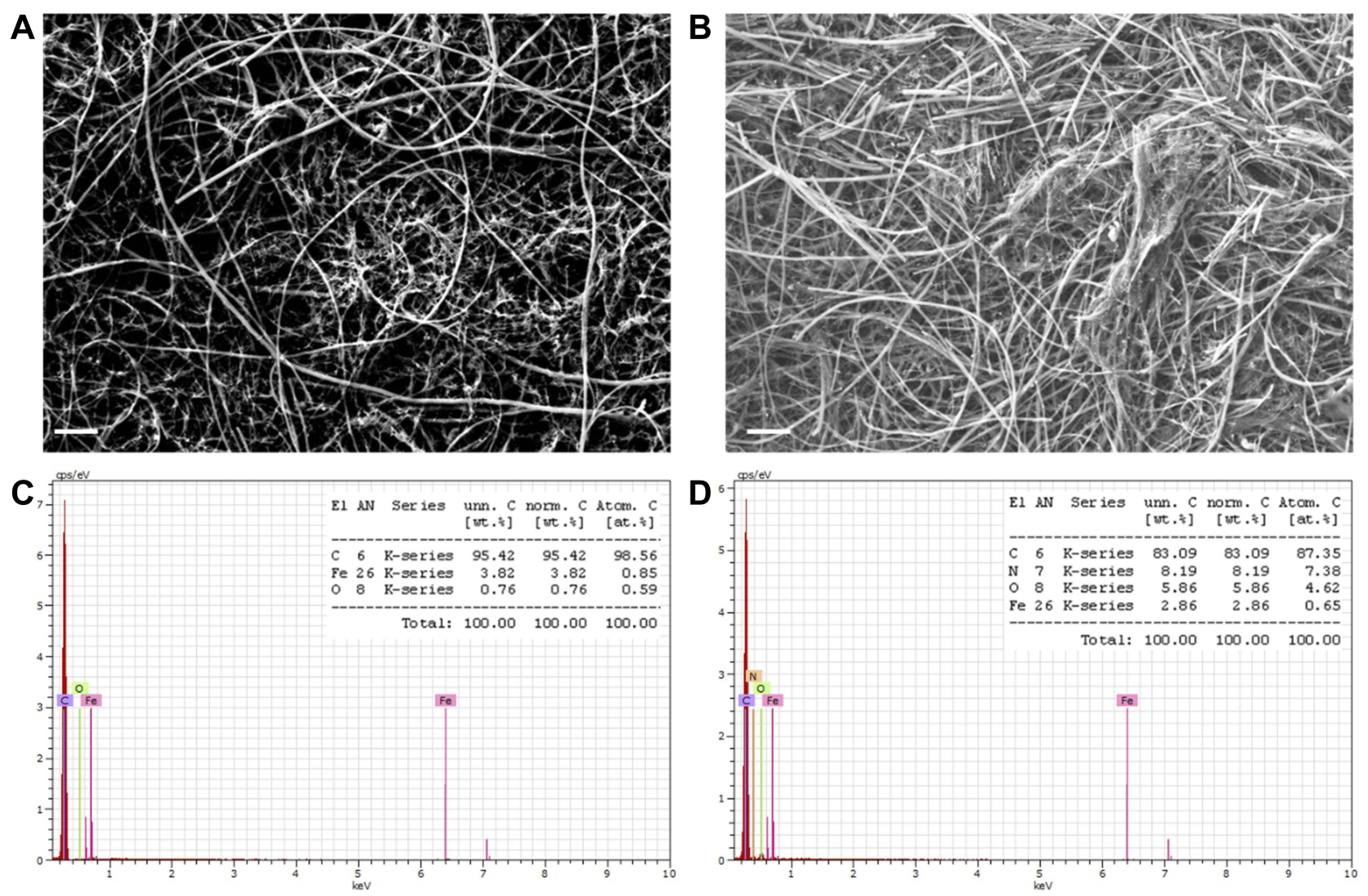

Figure I Scanning electron microscopy of the pristine buckypaper (BP) surface (A) and of the PAMAM-coated (PAM-BP) surface (B). The lower conductivity of the sample confirmed the effective coating with the PAMAM dendrimer. Scale bar: I $\mu \mathrm{m}$. Energy-dispersive X-ray analysis of the pristine BP (C) and PAM-BP (D).
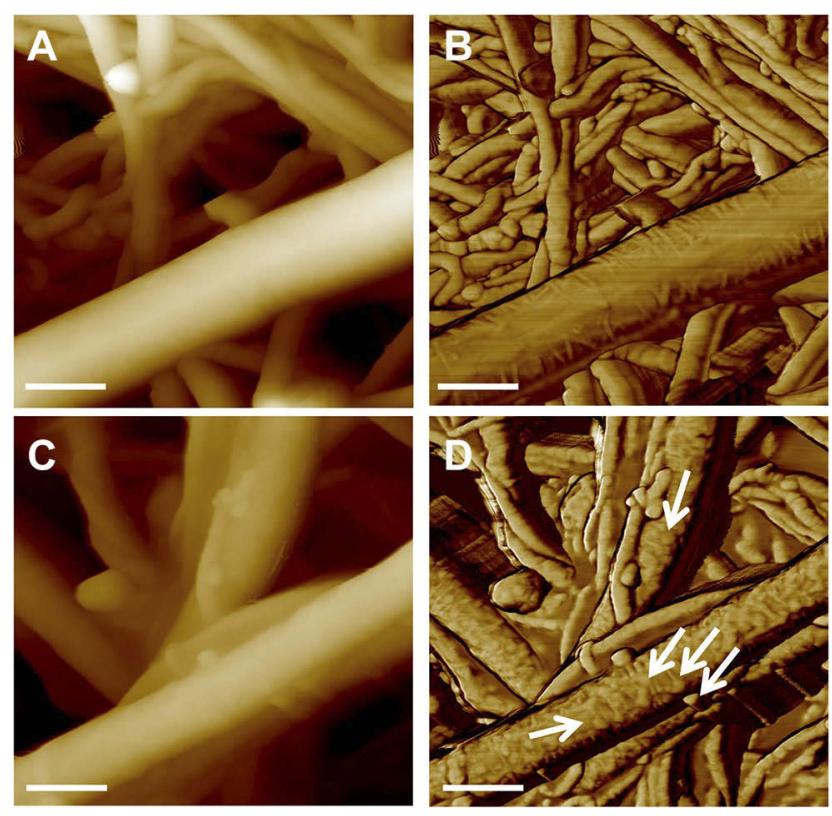

Figure 2 Height (left) and phase (right) channels by AFM measurements of the pristine buckypaper (A, B) and of the PAMAM-coated one (C, D). Arrows in panel D indicate the small bubble-like structures of the polymer coating the BP surface (D). In all panels, scale bar is $200 \mathrm{~nm}$. disorder of the structure and the G' band (at about $2690 \mathrm{~cm}^{-1}$ ) generally assigned to a second-order contribution. ${ }^{19}$ However, after PAMAM absorption, some specific spectral changes were observed (magnifications in Figure 3B-D). In Figure 3B, a relevant increase in the Raman intensity appears at about $2900 \mathrm{~cm}^{-1}$. This has been actually identified as the $\mathrm{CH}$ stretching region of the amine-terminated PAMAM G0 dendrimer, both by Raman ${ }^{20}$ and $\mathrm{IR}^{21}$ spectroscopy, so that the effect can be associated to an added contribution coming from the functionalization. Similarly, a remarkable increase of the intensity in the central spectral region is observable in Figure 3C, combined with a distinct new peak at about $1180 \mathrm{~cm}^{-1}$, and new bands at about 489 , 648 and $712 \mathrm{~cm}^{-1}$ also rise in the low wavenumber region, that is reported in Figure 3D. We assign all of these additional contributions (indicated by black arrows in the figures) to the stable interaction between the PAMAM dendrimer and the CNTs of the BP. This interpretation is confirmed by the red shift of the graphitic $\mathrm{G}$ band after the functionalization (data not shown), as the peak is broadened and displaced of about 

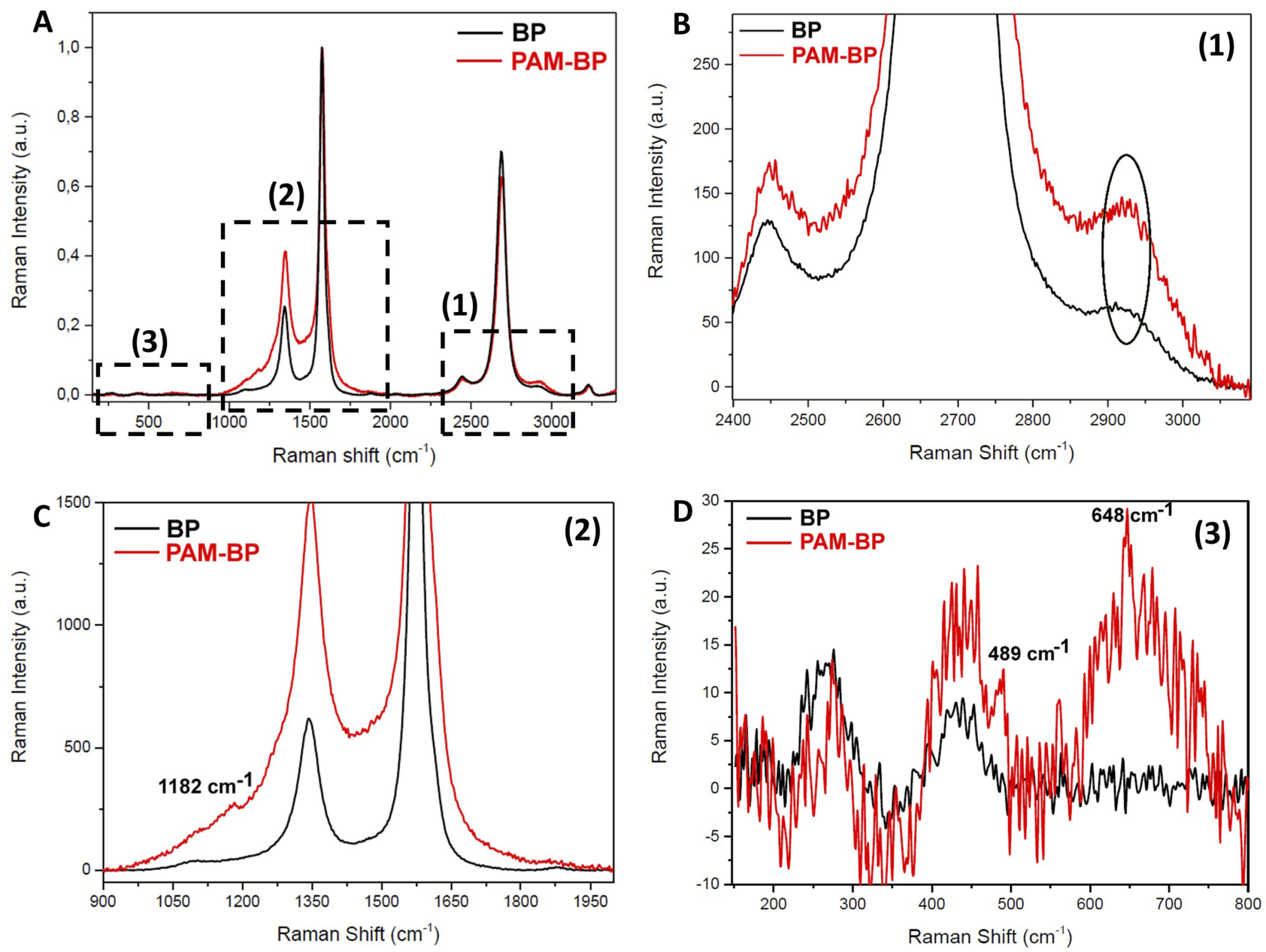

Figure 3 Raman spectra collected on BP (black curve) and PAM-BP (red curve) (A-D). Magnification of the high wavenumber spectral region (I; B); magnification of the central spectral region (2; C); and magnification of the low shift region (3; D).

$3 \mathrm{~cm}^{-1}$ as generally found when the surface of CNTs is modified by molecular labeling. ${ }^{22,23}$

\section{The PETG 3D-Printed Multi-Chamber Device Is Biocompatible}

The 3D-printed PETG device demonstrated a high robustness during the assembling and disassembling of the various components (i.e., rings, BPs, screws, etc.) even after repeated experiments. Moreover, PETG is a biocompatible material that displayed no cytotoxicity after prolonged cell culture. During cell culture, we noticed a slight reduction of the level of the culture medium although the device was kept covered by the custom 3D-printed cap (Figure 4) and no leakage from the device was observed. We cannot exclude that the higher hydrophilicity of PAM-BP allowed the support to absorb small amount of culture medium. In a typical set up, after 1, 3 and 7 days the 3D-printed multi- chamber device was opened, the BPs were removed and processed following different protocols.

To assess the kinetics of cell attachment and proliferation on the BP surface (both BP and PAM-BP), HEK-293T and Saos-2 cells were cultured for up to 7 days. To investigate biodegradability, osteoclasts were cultured on the same substrates for 3 days. By BCA assay, we evaluated the amount of cells grown on these substrates. For HEK-293T and Saos-2 cells seeded on pristine BP, the protein concentration after 1, 3 and 7 days was lower than the control sample (consisting in the same amount of cells seeded onto the BPs). This suggested that BP was not the ideal substrate for cells to grow or that BP was particularly cytotoxic at least in the initial phases (Figure 5). This behavior was remarkably different for PAM$\mathrm{BP}$ (compared to pristine $\mathrm{BP}$ ) as the number of cells grown on the latter substrate was significantly higher after the third day. After 3 days of culture, only cells grown on PAM-BP showed a slightly higher proliferation compared to control, although 

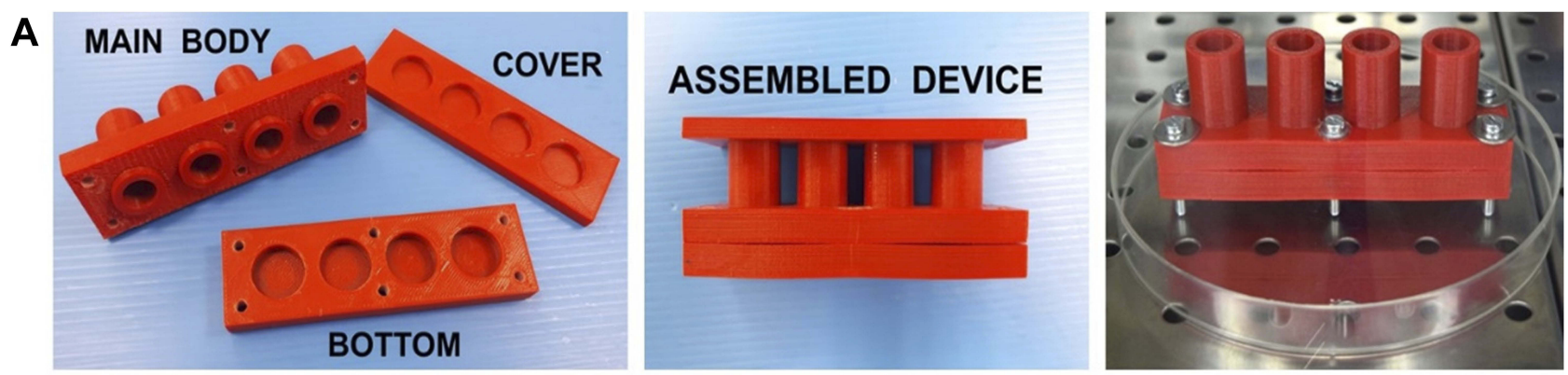

B
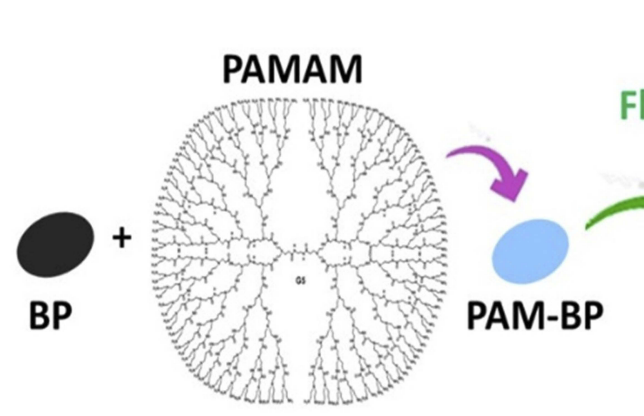

Fluorescent miRNA

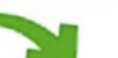

\section{PAM-BP-miRNA}

Fluorescent BP
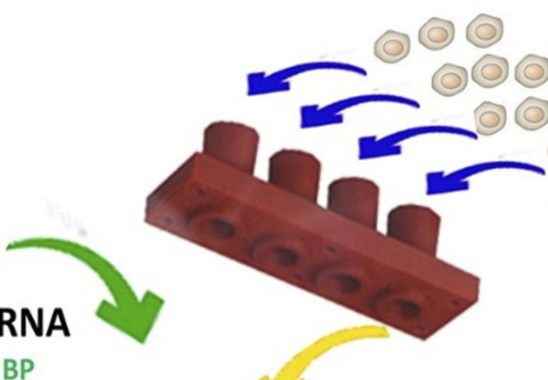

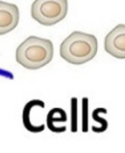

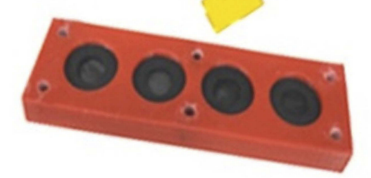

Figure 4 (A) 3D-printed multi-chamber device in its open and assembled configuration. (B) Procedure to obtain PAM-BP and assembling of the 3D-printed device.

the differences were not statistically significant. After 7 days, the cell growth on PAM-BP was significantly higher than that on BP (Figure 5). For Saos-2 and HEK-293T cells, the growth after 7 days was similar, suggesting that cells need more time to adapt and grow on the BP substrate. Moreover, the protein concentration that we measured for osteoclast cultures was

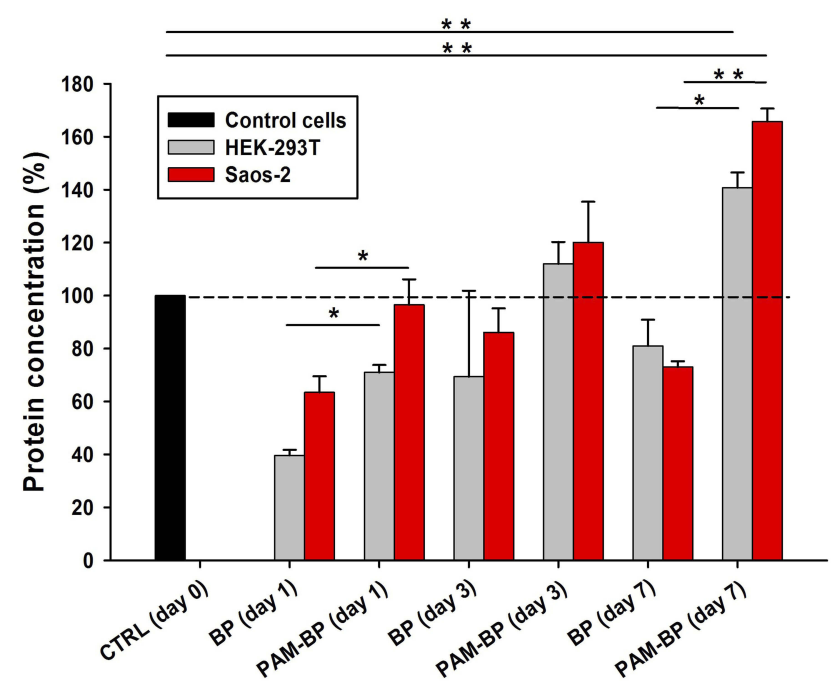

Figure $5 \mathrm{BCA}$ protein assay to monitor cell growth on buckypapers (BP and PAMBP). HEK-293T and Saos-2 cells have been cultured for up to 7 days and characterized at I, 3, and 7 days after seeding. $* \mathrm{P}<0.05$ and $* * \mathrm{P}<0.005$. slightly lower compared to that observed for HEK-293T and Saos-2 (Supplementary Figure S2), suggesting that BP and PAM-BP are not ideal substrates for osteoclast precursors to proliferate and differentiate. In any case, these measurements indicated that at least 7 days on PAM-BP were necessary for cells to adapt to buckypaper and start proliferating at a density similar to the initial condition (day 0 ). This behavior was not observed for pristine BP, suggesting that PAMAM coating was beneficial for a faster proliferation of cells.

\section{The 3D-Printed Multi-Chamber Device Allows To Culture Cells On BP}

In order to visualize the cell layer grown on BP at different time points, we had to disassemble the device at regular intervals, as its configuration coupled to the black surface of BP did not allow us to observe cells during the culture by classical microscopy methods (i.e., fluorescence or confocal). Therefore, we analyzed BPs (BP and PAM-BP) by electron microscopy techniques at the end of the culture.

After 3 days, SEM images showed the presence of cells attached on the BP surface without significant differences between BP and PAM-BP (Figure 6A, panels A1-A2). Although we noticed that cells cultured on both substrates were suffering (i.e., we noticed the presence of rounded 
cells) and dying (i.e., we observed some broken cells on BP substrate), cells cultured on BP and PAM-BP were equally abundant (Figure 6A, panels A1-A2). On the contrary, after incubation for 7 days, we observed a significantly higher number of cells in PAM-BP compared to BP alone (Figure 6A, panels A3-A4). The clusters of cells grown on the smoother PAM-BP surface suggested that PAM-BP is a more suitable environment for cell culture, likely due to PAMAM coating.

With osteosarcoma Saos-2 cells, we observed a similar behavior. In fact, cells cultured on BP for 7 days displayed a squashed appearance (Figure 6B, panel B1), whereas those cultured on PAM-BP were well defined and adherent to the support (Figure 6B, panel B2).

Finally, in order to assess the usefulness of PAMAMcoated BP for biomedical applications and preliminarily evaluate their biodegradability, we studied the ability of osteoclasts to adhere on BP and PAM-BP. Osteoclasts are large, multinucleated, motile cells that are formed by the fusion of hematopoietic cells of the monocyte/macrophage lineage. ${ }^{24,25}$ We evaluated by SEM imaging their ability to degrade the BP substrate. After culturing osteoclasts for 3 days, their morphology appeared completely squashed on the BP surface (Figure 6B, panel C1) underlining once again the incompatibility and cytotoxicity of the pristine BP toward this cell line. Again, PAMAM coating allowed to obtain a favorable substrate for cell culture, although cells showed a rounded appearance (Figure 6B, panel C2). Unfortunately, SEM images did not allow us to obtain a conclusive information about the ability of osteoclasts to resorb the BP structure as no signs of degradation were visible.

A
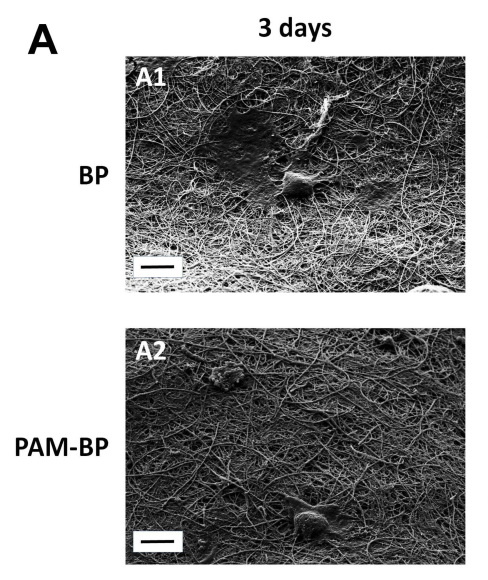

HEK-293T

\section{PAM-BP Allows Transfection And A Prolonged Release Of microRNAs Into Cells}

Owing to the ability of PAM-BP to allow the growth of human cells, we were aimed at assessing the ability of BP to transfect cells for up to 7 days. To this purpose, the PAM-BP support was incubated with a fluorescent oligonucleotide (i.e., FAM-pre-mir-503), a microRNA mimic that some of us have already employed in a previous paper $^{2}$ (Figure 7). Only the coating of BP with PAMAM allowed the BP to bind the fluorescent oligonucleotide, form a complex (PAM-BP-miRNA) and fluoresce under confocal microscopy (Figure 7A). In fact, BP alone was not able to bind the oligonucleotide by itself and this resulted in the absence of fluorescence and transfection (data not shown).

Confocal microscopy emphasized that PAM-BPmiRNA was fluorescent also in the absence of cells and that many focal planes were present (Figure 7). In line with proliferation assays, after 1 and 3 days of culture, the seeded cells were only partially transfected (Figure 7B-C), whereas after increasing the incubation time up to 7 days, cells were all transfected (Figure 7C).

To better understand the mechanism of transfection by PAM-BP, we prepared a fluorescently labeled dendrimer (PAMAM labeling with the Rhodamine-B dye), ${ }^{18}$ in order to examine the morphology of PAM-BP (Figure 8A). With this labeling procedure, we were able to observe RhoPAM-BP and investigate whether the transfection that we observed was due to polymer detachment or to BP (i.e., CNTs). In fact, it has been reported that CNTs can
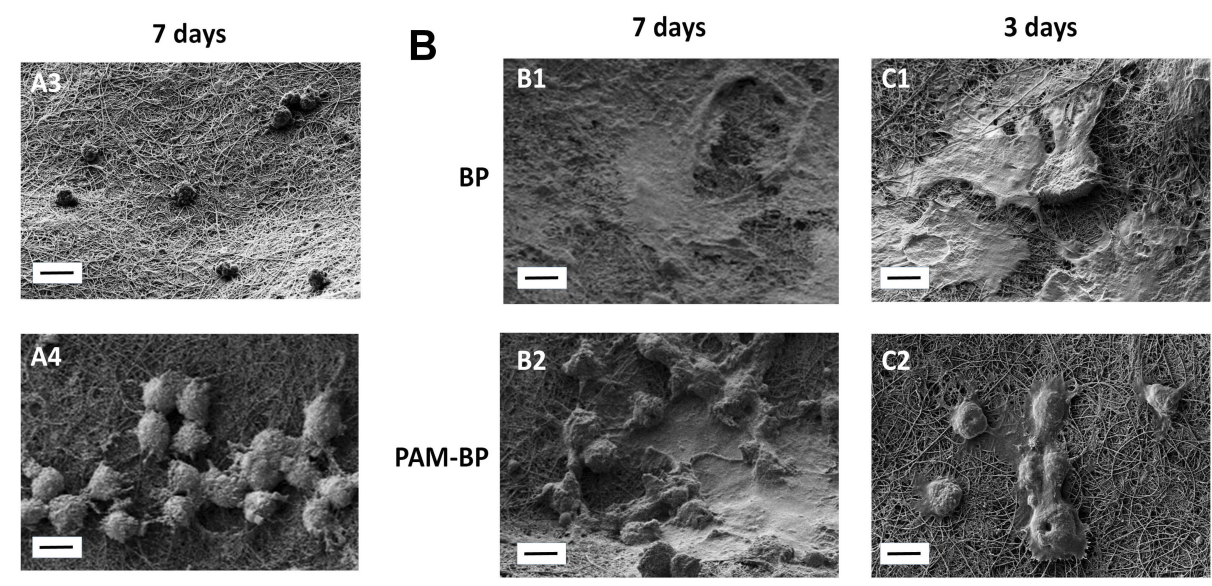

Saos-2

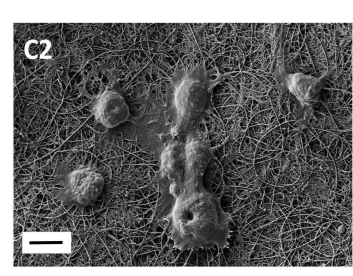

Osteoclasts

Figure 6 Scanning electron microscopy of HEK-293T (A), Saos-2 and osteoclast cells (B) grown on buckypapers at different time points ( 3 and 7 days). Scale bars are I0 $\mu$ m. 
A

Pristine BP

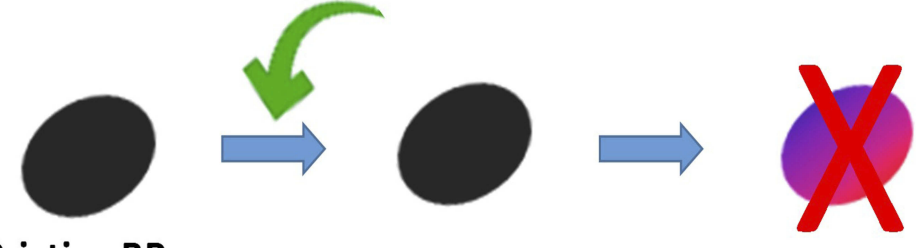

Incubation with a

fluorescent miRNA

NOT FLUORESCENT

Incubation with a

Incubation

with PAMAM

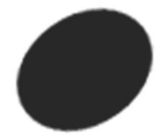

Pristine BP
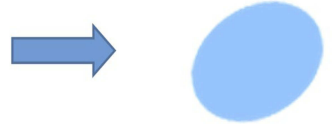

政
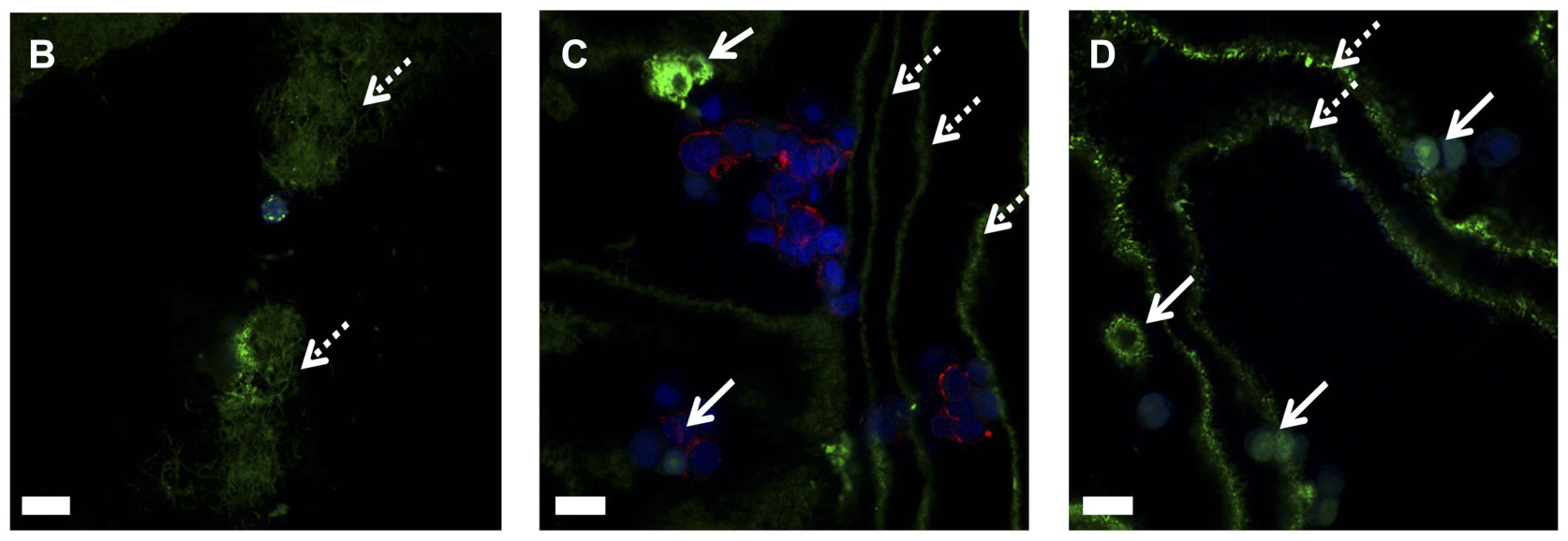

Figure 7 Preparation procedure for BP coating with PAMAM dendrimer and complexation with a fluorescent miRNA (A). Confocal microscopy of HEK-293T transfected for I day (B), 3 days (C) and 7 days (D) with PAM-BP functionalized with FAM-Pre-mir-503 mimic (400x magnification). The green lines (indicated by dashed white arrows) indicate different focal planes, while the intense green spots (indicated by white arrows) represent the transfected cells.

transfect cells also by piercing effect. ${ }^{2,26}$ With RhodamineB functionalization of PAMAM, we were able to observe the presence of red spots inside osteoclasts after transfection, suggesting that PAMAM might detach from the BP surface and enter into cells (Figure $8 \mathrm{~B}$ ). To confirm this hypothesis (i.e., the kinetics of release of the polymer absorbed to BP), we performed a TGA analysis on PAMBP kept in PBS for 1,3 and 7 days to simulate the culturing condition. We found that after one day of incubation the percentage of polymer absorbed onto BP significantly decreased from $9.37 \pm 1.07$ to $3.91 \pm 0.98$ and remained stable for up to 7 days without significant modulations (Figure 8C). This means that polymer-coated BP slowly releases PAMAM from the surface and that this polymer can enter into cell, likely conveying also miRNAs complexed to them. This behavior underlines the advantage of using PAMAM polymer in BP functionalization, as this polyamide has the double action of increasing cell attachment on BP and allowing the internalization of oligonucleotides into cells. This property is advantageous when looking for materials able to release slowly a drug in the surrounding environment.

The biodegradation of the substrates employed in biomedicine is a major issue, especially in regenerative medicine. Being osteoclasts the only cell type in the human body able to degrade (resorb) extracellular bone matrix, we performed a 

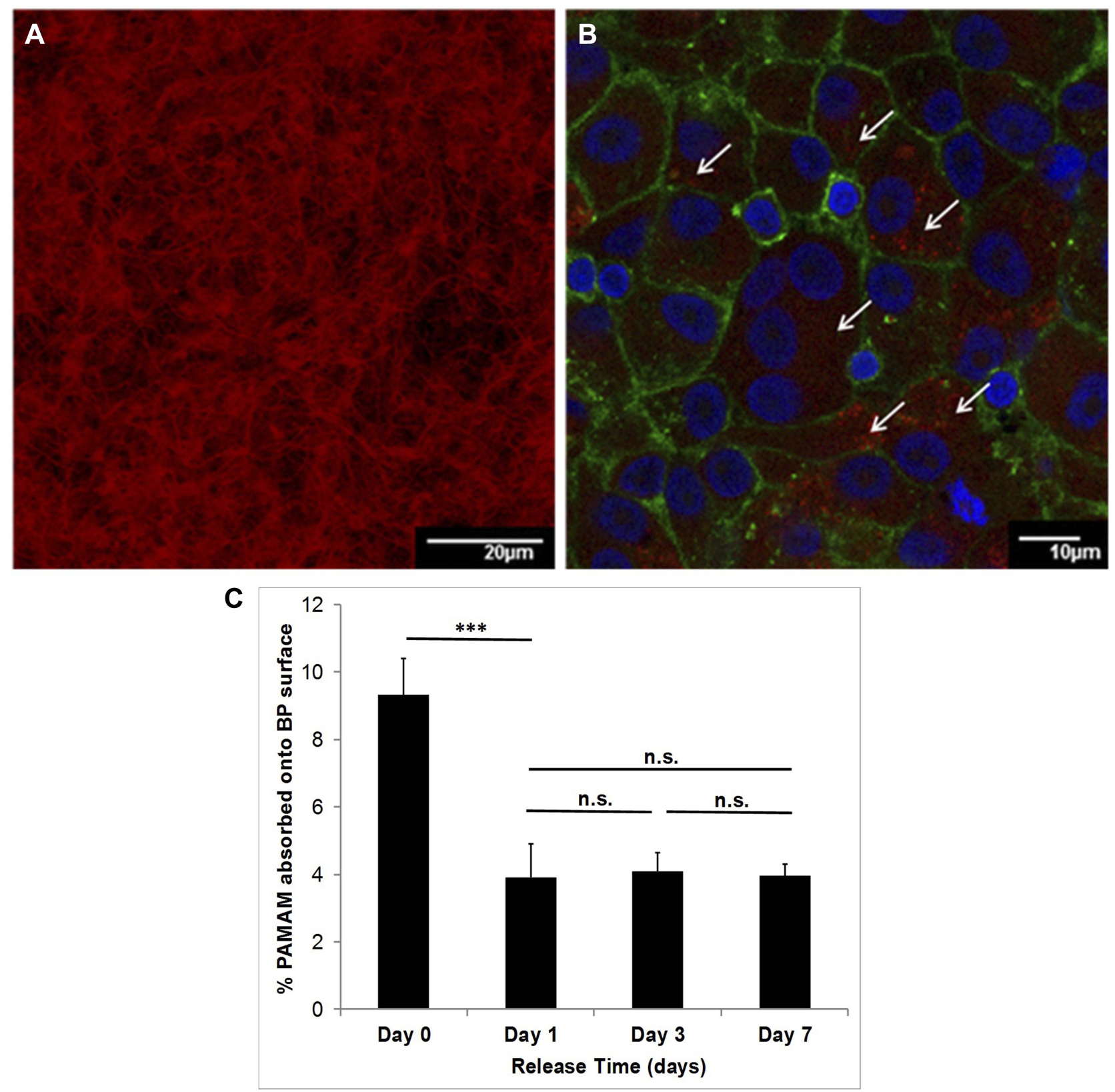

Figure 8 Confocal microscopy of the buckypaper incubated with PAMAM dendrimer labeled with rhodamine B isothiocyanate (I0\% w/w). The red filaments confirm the attachment of the polymer to the surface (A) (200x magnification). The osteoclasts membrane was stained with phalloidin-FITC and the multinucleated nuclei with Hoechst, which allows distinguishing the osteoclasts from their precursors (B). The red spots (indicated by white arrows) confirm the presence of PAMAM within the cells that is released progressively. Quantification of PAMAM absorbed onto BP surface (expressed as percentage in weight) $(* * *=p-v a l u e<0.00 \mathrm{I} ; \mathrm{n} . \mathrm{s}=\mathrm{not}$ significant) $(\mathbf{C})$.

pilot experiment by employing these cells to assess the PAMBP biodegradation. Osteoclasts were grown on BPs and the study of the F-actin morphology by phalloidin staining allowed us to analyze the osteoclast membrane reorganization (Figure 8B). The absence of sealing zones, specific actin-rich adhesion structures by which the osteoclast attaches tightly to the bone matrix ${ }^{27}$ suggested that osteoclasts were unable to create a sealed compartment below their space, where BP degradation might occur. One of the possible explanations might be the intrinsic aspect of $\mathrm{BP}$ surface: a wide-mesh network that prevented the formation of a sealed area under the cells, preventing resorption.

\section{Discussion}

In this work, we demonstrated that the coupling of 3D printing technology (i.e., fabrication of custom devices) to the nanomedicine field (i.e., cell culture on nanostructured substrates) is a strategy that allowed to obtain customized 
models used to test in vitro the ability of cells to grow in so far unimaginable substrates, such as CNTs BP. BPs are highly hydrophobic and not suited to culture cells unless properly functionalized. The advantage of using BP in the biomedical field is linked to its properties (mainly high resistance and robustness) that could facilitate its use, for example, in tissue regeneration.

We recently demonstrated that the coating of CNTs with poly-amidoamine dendrimers (PAMAM, G5) is a useful strategy to impart hydrophilicity to surfaces, increase adhesiveness and cell adhesion, and exploit additional functions such as transfection of microRNAs. ${ }^{2,18}$

Therefore, in order to expand the repertoire of substrates to employ for cell proliferation and transfection, we assessed the properties of commercial BP coated with PAMAM. To our knowledge, this is the first report of a 2D-nanostructured material (i.e., the BP) coated with a polymer able to facilitate the proliferation of cells for long periods and, at the same time, to deliver a cargo with modulatory activities (such as miRNAs). Although we are aware that further experiments are needed (i.e., in vivo experiments) to prove that these BP could be employed in many biomedical fields, we devised the first nanostructured substrate containing cell layers and able to be manipulated easily (i.e., to be used for potentially implantable devices). In fact, we have previously demonstrated that CNTs coated with PAMAM showed a reduced toxicity and we verified that also coated BP was not cytotoxic. The proliferation increase observed after 7 days indicated that cell growth on these substrates is possible and achievable.

We noticed that depending on cell type, these supports allow a different cell growth. HEK-293T and the osteogenic Saos-2 cells grow quite rapidly on these modified substrates, whereas osteoclasts adhere and grow slower than HEK-293T and Saos-2. This is an important point to study further and to consider when deciding to prepare devices for tissue regeneration applications.

\section{Conclusion}

One of the disadvantages of this version of the multi-chamber device is the fact that it is not transparent to light. Therefore, the observation of cells at different time points is practically not possible, unless the apparatus is disassembled.

We suggest that to solve some of the "black boxes" that we found during cell culture, one of the future strategy would be to 3D print the device by using clear/transparent PETG filaments or other transparent materials.

Moreover, we also demonstrated that PAM-BP is able to release the absorbed polymer (PAMAM) and allow a controlled transfection of miRNAs into cells. This encouraged us to think that PAMAM-coated BP might be used also for controlled delivery of drugs both in vitro and in vivo and exploit innovative therapeutic strategies.

Finally, we think that in the near future we will assist to a progressive adoption of 3D printing technology not only in the biomedical field (where it is currently widely used) but also in other nanotechnology fields.

\section{Acknowledgments}

The authors thank Prof. Antonio D'Alessandro (Director of the Center for The Nanotechnology Applied to Engineering of La Sapienza - CNIS) and Prof. Federico Bordi for useful discussions and for the access to CNIS SNN-Lab for electron microscopy. This work received funding from the Italian Ministry of Health (Progetto Ricerca Finalizzata PE-2011-02347026).

This work was presented at the Nanoscience and Nanotechnology conference organized by the NEXT Nanotechnology group at INFN - LNF (Rome, Italy), October 15-18, 2019.

\section{Disclosure}

The authors report no conflicts of interest in this work.

\section{References}

1. Masotti A, Miller MR, Celluzzi A, et al. Regulation of angiogenesis through the efficient delivery of microRNAs into endothelial cells using polyamine-coated carbon nanotubes. Nanomedicine. 2016;12 (6):1511-1522. doi:10.1016/j.nano.2016.02.017

2. Celluzzi A, Paolini A, D’Oria V, et al. Biophysical and biological contributions of polyamine-coated carbon nanotubes and bidimensional buckypapers in the delivery of miRNAs to human cells. Int $J$ Nanomedicine. 2017;13:1-18. doi:10.2147/IJN.S144155

3. Cui HF, Vashist SK, Al-Rubeaan K, Luong JH, Sheu FS. Interfacing carbon nanotubes with living mammalian cells and cytotoxicity issues. Chem Res Toxicol. 2010;23(7):1131-1147. doi:10.1021/ tx100050h.

4. Yang ST, Luo J, Zhou Q, Wang H. Pharmacokinetics, metabolism and toxicity of carbon nanotubes for biomedical purposes. Theranostics. 2012;2(3):271-282. doi:10.7150/thno.3618

5. Price RL, Waid MC, Haberstroh KM, Webster TJ. Selective bone cell adhesion on formulations containing carbon nanofibers. Biomaterials. 2003;24(11):1877-1887. doi:10.1016/S0142-9612(02)00609-9

6. Hu L, Hecht DS, Grüner G. Percolation in transparent and conducting carbon nanotube networks. Nano Lett. 2004;4(12):2513-2517. doi: $10.1021 / \mathrm{n} 1048435 \mathrm{y}$

7. Correa-Duarte MA, Wagner N, Rojas-Chapana J, Morsczeck C, Thie M, Giersig M. Fabrication and biocompatibility of carbon nanotubebased 3D networks as scaffolds for cell seeding and growth. Nano Lett. 2004;4:2233-2236. doi:10.1021/n1048574f

8. Dinicola S, Masiello MG, Proietti S, et al. Multiwalled carbon nanotube buckypaper induces cell cycle arrest and apoptosis in human leukemia cell lines through modulation of AKT and MAPK signaling pathways. Toxicol In Vitro. 2015;29(7):1298-1308. doi:10.1016/j. tiv.2015.05.006 
9. Meng J, Song L, Meng J, et al. Using single-walled carbon nanotubes nonwoven films as scaffolds to enhance long-term cell proliferation in vitro. J Biomed Mater Res A. 2006;79(2):298-306. doi:10.1002/jbm. a.30787

10. Stevens MM, George JH. Exploring and engineering the cell surface interface. Science. 2005;310(5751):1135-1138. doi:10.1126/science. 1106587

11. MacDonald RA, Laurenzi BF, Viswanathan G, Ajayan PM, Stegemann JP. Collagen-carbon nanotube composite materials as scaffolds in tissue engineering. J Biomed Mater Res A. 2005;74 (3):489-496. doi:10.1002/jbm.a.30386

12. Gross BC, Erkal JL, Lockwood SY, Chen C, Spence DM. Evaluation of 3D printing and its potential impact on biotechnology and the chemical sciences. Anal Chem. 2014;86(7):3240-3253. doi:10.1021/ac403397r

13. Goldstein TA, Epstein CJ, Schwartz J, et al. Feasibility of bioprinting with a modified desktop 3D printer. Tissue Eng Part C Methods. 2016;22(12):1071-1076. doi:10.1089/ten.TEC.2016.0286

14. Baden T, Chagas AM, Gage GJ, Marzullo TC, Prieto-Godino LL, Euler T. Open Labware: 3-D printing your own lab equipment. PLoS Biol. 2015;13(3):e1002086. doi:10.1371/journal.pbio.1002086

15. Wijnen B, Hunt EJ, Anzalone GC, Pearce JM. Open-source syringe pump library. PLOS ONE. 2014;9(9):e107216. doi:10.1371/journal. pone.0107216

16. Zhang YS, Ribas J, Nadhman A, et al. A cost-effective fluorescence mini-microscope for biomedical applications. Lab Chip. 2015;15 (18):3661-3669. doi:10.1039/c5lc00666j

17. Hernandez Vera R, Schwan E, Fatsis-Kavalopoulos N, Kreuger JA. Modular and affordable time-lapse imaging and incubation system based on 3D-printed Parts, A smartphone, and off-the-shelf electronics. PLoS One. 2016;11(12):e0167583. doi:10.1371/journal. pone. 0167583

18. Paolini A, Leoni L, Giannicchi I, et al. MicroRNAs delivery into human cells grown on 3D-printed PLA scaffolds coated with a novel fluorescent PAMAM dendrimer for biomedical applications. Sci Rep. 2018;8(1):13888-018-32258-9. doi:10.1038/s41598-018-32258-9.
19. Bokobza L, Bruneel JL, Couzi M. Raman spectra of carbon-based materials (from graphite to carbon black) and of some silicone composites. C. 2015;1(1):77-94.

20. Davis AP, Ma G, Allen HC. Surface vibrational sum frequency and Raman studies of PAMAM G0, G1 and acylated PAMAM G0 dendrimers. Anal Chim Acta. 2003;496(1):117-131. doi:10.1016/S00032670(02)01375-2

21. Manna A, Imae T, Aoi K, Okada M, Yogo T. Synthesis of dendrimerpassivated noble metal nanoparticles in a polar medium: comparison of size between silver and gold particles. Chem Mater. 2001;13 (5):1674-1681. doi:10.1021/cm000416b

22. Visentin S, Barbero N, Bertani FR, et al. Multivariate analysis applied to Raman mapping of dye-functionalized carbon nanotubes: a novel approach to support the rational design of functional nanostructures. Analyst. 2015;140(16):5754-5763. doi:10.1039/C5AN00820D

23. Marković Z, Jovanović S, Kleut D, et al. Comparative study on modification of single wall carbon nanotubes by sodium dodecylbenzene sulfonate and melamine sulfonate superplasticiser. Appl Surf Sci. 2009;255(12):6359-6366. doi:10.1016/j.apsusc.2009.02.016

24. Boyle WJ, Simonet WS, Lacey DL. Osteoclast differentiation and activation. Nature. 2003;423(6937):337-342. doi:10.1038/nature01658

25. Itzstein C, Coxon FP, Rogers MJ. The regulation of osteoclast function and bone resorption by small GTPases. Small GTPases. 2011;2 (3):117-130. doi:10.4161/sgtp.2.3.16453

26. Kobler C, Poulsen SS, Saber AT, et al. Time-dependent subcellular distribution and effects of carbon nanotubes in lungs of mice. PLoS ONE. 2015;10(1):e0116481. doi:10.1371/journal.pone.0116481

27. Luxenburg C, Geblinger D, Klein E, et al. The architecture of the adhesive apparatus of cultured osteoclasts: from podosome formation to sealing zone assembly. PLoS ONE. 2007;2(1):e179. doi:10.1371/ journal.pone.0000179
International Journal of Nanomedicine

\section{Publish your work in this journal}

The International Journal of Nanomedicine is an international, peerreviewed journal focusing on the application of nanotechnology in diagnostics, therapeutics, and drug delivery systems throughout the biomedical field. This journal is indexed on PubMed Central, MedLine, CAS, SciSearch ${ }^{\mathbb{R}}$, Current Contents ${ }^{\mathbb{R}} /$ Clinical Medicine,

\section{Dovepress}

Journal Citation Reports/Science Edition, EMBase, Scopus and the Elsevier Bibliographic databases. The manuscript management system is completely online and includes a very quick and fair peer-review system, which is all easy to use. Visit http://www.dovepress.com/ testimonials.php to read real quotes from published authors. 